\title{
Limited Liability Contracts between Principal and Agent*
}

\begin{abstract}
DAVID SAPPINGTON ${ }^{\dagger}$
Department of Economics, The University of Michigan, Ann Arbor, Michigan 48109

Received April 17, 1980; revised September 30, 1981

The optimal strategy of the principal is examined in an environment where there are (ex post) limitations on the maximum penalty that can be imposed on a risk neutral agent. Contrary to the case in which such limitations are not imposed, it is in the principal's interest to deliberately forego the opportunity to induce socially efficient behavior, and to instead design a contract that induces the agent to realize an efficient outcome only in the most productive state of nature and (perhaps) in certain very unproductive states. The properties of the contract are examined in detail. Journal of Economic Literature Classification Numbers: 022, 026, 610.
\end{abstract}

\section{INTRODUCTION}

In the past decade, the principal-agent model has received considerable recognition as an important analytic device in the study of incentive schemes and contracts among economic agents. (See, for example, the works of Ross [18, 19], Harris and Raviv [11], Holmstrom [13], Hurwicz and Shapiro [14], and Shavell [23].) The particular version of the model that is explored in this paper assumes that the principal and agent consummate an agreement at a point in time when they share symmetric beliefs about the probability distribution of a random state of nature, $\theta$. The realization of $\theta$, which is subsequently observed by the risk-neutral agent (alone) before choosing his (unobservable) level of effort, affects the productivity of such effort.

Harris and Raviv [10] show that in this setting, the self-interested principal can and will design a contract that induces an outcome in every state

Partial financial support for this research through a grant from the Sloan Foundation to the Princeton University Economics Department is gratefully acknowledged. The author wishes to express special thanks to Robert D. Willig for his patient guidance, stimulating discussions, and insightful comments. The suggestions and direction provided by Gregory C. Chow and Steven C. Salop in the earlier stages of this research are also greatly appreciated. In addition, I am grateful to Edward J. Green, John C. Panzar, Andrew Postlewaite, and an anonymous referee for their helpful comments. The author assumes sole responsibility for any remaining errors.

${ }^{\dagger}$ Present address: Department of Economics, University of Pennsylvania, Philadelphia, Pa. 19104. 
of nature that is Pareto efficient. However, such a contract is necessarily optimal for the principal only when institutions exist which gurantee that the agent does not breach the contract after he observe $\theta$, no matter how debilitating compliance may be for the agent in that state. In the absence of such institutions, the contract that the principal will design is likely to be of a very different form. ${ }^{1}$

It is the purpose of this paper to examine the properties of the contract that will emerge between principal and risk-neutral agent when limits are imposed on the maximum loss that the agent can be forced to bear as a consequence of contracting with the principal. Contracts which incorporate such limits on the ex post liability of the agent will be referred to as limited liability contracts.

Contracts in which the liability of one or more parties is explicitly limited are very common in practice. Bankruptcy clauses, statements of conditions under which breach of contract is permissible, and provisions in corporate charters which limit the liability of each stockholder to the value of his shares are all examples of limited liability clauses. Contracts which contain such clauses are particularly conspicuous in practice when: (1) information about risk is incomplete or cannot be attained at the same cost by all parties to the contract (see [1]), (2) social concerns warrant subsidies for participation in certain activities (such as corporate investment) (see [17, p. $177 \mid$ ), and (3) paternalism and/or equity consideration mandate riskspreading or the guarantee of a subsistence level of "well-being" for each member of society (see for example, $[1,4]$ ).

For the purposes of illustration and analytic convenience, this paper will initially focus on the special case of limited (zero) liability contracts in which the agent has the legal right to disassociate himself from the principal without penalty after observing the state of nature. It is shown that in this environment, the self-interested principal will design a contract that in all states except the one in which the agent is most productive, and perhaps in certain very unproductive states, induces outcomes that are ex post Pareto inefficient. First, though, the interaction between principal and agent that is analyzed in this paper is stated more precisely in Section 2 . Section 2 also contains a brief comparison of the model considered here to others in the literature.

After the principal's choicc of a zero-liability contract is described in detail and shown to induce inefficient outcomes in all but a selected few states of nature (see Section 3), an intuitive explanation of these results is offercd in Section 4. Then, in Section 5, more general forms of limited liability contracts are analyzed. The qualitative properties of the zero-

\footnotetext{
' A number of authors, including Holmstrom [13] and Lewis [15], have noted that liability limitations may be important to consider in this context.
} 
liability cuntract are shown to be unaltered by the introduction of this greater generality. Finally, conclusions are drawn in Section 6.

\section{Statement of the Model}

In the model analyzed here, the principal owns a productive technology that requires as an input the (action or) effort, $a$, of the risk-neutral agent. This effort, together with the realization of the random state of nature, $\theta$, determine the value of output produced, $x$, according to the relationship $x=X(a, \theta)$.

When a contract is agreed upon, both the principal and agent know the distribution of $\theta$. It is only later that the agent (alone) observes the actual realization of $\theta$, and then selects an (unobservable) action. The fact that the principal can observe neither a nor $\theta$ mandates that the contract specify payments to the agent, $S$, as a function of $x$ only. Any such contract will only be accepted by the agent if it offers him a level of expected utility that exceeds his reservation utility level, $U^{0}$, the magnitude of which is known to the principal.

In order to isolate the effects of liability limitations, all of the potential risk-sharing attributes of any contract are eliminated by the assumption that both the principal and agent are risk-neutral. It is assumed that the principal's objective is to maximize the expected value of $x-S(x)$, and that the agent's utility function is given by $U^{A}(x, S)=S-W(x, \theta)$, where $W(x, \theta)$ is the dollar value of the disutility to the agent of producing $x$ in state $\theta .^{2}$

Letting alphabetic subscripts indicate partial derivatives, $W(\cdot, \cdot)$ is assumed throughout to be characterized by all but the last of the following properties. $W(\cdot, \cdot)$ is also assumed to be characterized by Property (6) in the proof of Proposition 2.

$$
\begin{aligned}
& \text { Property (1): } W_{\theta}(x, \theta) \leqslant 0 ; \\
& \text { Property (2): } W_{x}(x, \theta) \geqslant 0 ; \\
& \text { Property (3): } W_{x \theta}(x, \theta) \leqslant 0 ; \\
& \text { Property (4): } W_{x x}(x, \theta) \geqslant 0 ; \\
& \text { Property (5): } W_{x}\left(0, \theta_{n}\right)<1 ; \\
& \text { Property (6): } W_{x x \theta}(x, \theta) \leqslant 0 ;
\end{aligned}
$$

where $0<\theta_{1}<\cdots<\theta_{n}$.

${ }^{2}$ More explicitly, following $[13,14]$, the agent's utility function is assumed to be separable in effort and income, so that $U^{\prime A}(a, S)=S-V(a)$. Then, if $V^{\prime}(a)>0$, and if $a=g(x, \theta)$ is the minimum amount of effort required to produce $x$ in state $\theta$, the stated form of the agent's utility function follows if $W(x, \theta)=V(g(x, \theta))$. 
These properties hold for all values of $\theta$ and all non-negative values of $x$. The inequalities are strict whenever $x$ is strictly positive. ${ }^{3}$

Properties (1) and (3) indicate that $\theta$ can be thought of as a productivity parameter, where higher values of $\theta$ correspond to states in which the agent is more productive and in which additional output is less onerous to produce. Properties (2) and (4) indicate that in every state of nature, the marginal disutility of effort to the agent is positive and increasing. (Property (6) states that the agent's marginal disutility of effort increases less rapidly in higher states of nature.) Property (5) simply ensures the existence of a non-trivial solution to the problem at hand. It states that there is some strictly positive level of output that can be produced by the agent in the most productive state of nature without incurring a level of disutility which exceeds the value of that output to the principal.

In what follows, outcomes of the interaction between principal and agent will be referred to as either efficient of inefficient. An efficient outcome is one that is ex post Pareto efficient in the particular state of nature that prevails, and an inefficient outcome is any one that is not efficient. The value of output, $x_{i}^{*}$, that is efficient in state $\theta_{i}$ is the one at which the agent's marginal disutility from generating an additional unit of output coincides with the principal's valuation of such output, i.e., $W_{x}\left(x_{i}^{*}, \theta_{i}\right)=1$.

Contracts will similarly be classified in the ensuing discussion according to whether or not they are "first-best." A first-best contract is one that results in the realization of an efficient outcome whatever the state of nature that is ultimately realized. A first-best contract, by definition, maximizes the expected total surplus from production. The phrase "first-best" is meant to suggest a comparison with the situation in which the state of nature can be observed by the principal so that a forcing contract (see [10]) can be designed to ensure that an efficient outcome is realized in every state and that the agent receives no more than his reservation level of utility in any state.

Harris and Raviv $[10]$ have shown that under the conditions of asymmetric information considered here (and under even more general conditions,

${ }^{3}$ It should be noted here that for expositional convenience. Properties 1 through 6 are stated as if the distribution of $\theta$ were continuous. This distribution is, however. assumed to be discrete throughout the ensuing analysis. The assumption of a discrete distribution facilitates the comparative statics analysis in Section 4.

The assumption also adds strength to the conclusion that the optimal limited liability contract is not first-best. Sappington $|21|$ has shown that the set of contracts between principal and agent that are first-best when $\theta$ has a continuous distribution is a proper subset of the set of first-best contracts when the distribution of $\theta$ is discrete. Therefore, a finding that the principal will not offer the agent a limited liability contract that is first-best when $\theta$ has a continuous distribution would not necessarily imply that the same is true when $\theta$ has a discrete distribution. 
including the possibility of risk aversion on the part of the principal), the contract that maximizes the expected utility of the principal in the absence of liability restrictions is a first-best contract of the form $S(x)=x-k$. Here, $k$ is any expected surplus from efficient production in excess of that required by the agent in order contract with the principal, i.e., $k \equiv \sum_{i=1}^{n} p_{i}\left[x_{i}^{*}-\right.$ $\left.W\left(x_{i}^{*}, \theta_{i}\right)\right]-U^{0}$, where $p_{i}(>0 \forall i)$ is the probability that $\theta_{i}$ will be realized. Under this contract, the agent pays $k$ to the principal whatever the state of nature, and in return, retains the entire (efficient) value of output that he chooses to produce.

Although this contract promises the risk-neutral agent his reservation level of expected utility, when certain (of the lower) states of nature occur the agent can do no better under this contract than suffer a loss in utility below the level achieved in autarky (i.e., if he had not contracted with the principal at all). In these states, the agent would like to breach the contract, but the institutions that are assumed to exist throughout the principal-agent literature prevent him from doing so. ${ }^{4}$

For one or more of the reasons noted in the introduction, such institutions may, in reality, not exist. Hence, it is of interest to determine how the feasible contract that maximizes the principal's expected utility is altered by the absence of institutions that bind the agent to any and all ex ante agreements. In particular, suppose that there is a maximum penalty (i.e., a minimum value for $S(\cdot)$ ) that can be imposed upon the agent regardless of the outcome of his action. In such a situation, the principal's problem can be formulated as follows: ${ }^{5}$

$$
\left.\underset{S(x)}{\operatorname{Maximize}} \quad E_{\theta}\{X(a\langle S, \theta\rangle, \theta)-S \mid X(\cdot)]\right\}
$$

${ }^{4}$ One such "institution" may simply be the requirement that the agent pay the lump sum, $k$ (e.g., post bond), at the time when the contract is signed. This institution may not be feasible, however, when the agent's total resources are less than $k$ and when he cannot acquire income insurance.

Furthermore, because any insurance plan which yields a positive expected profit to a third party is also profitable for the principal (assuming this third party can observe neither $\theta$ nor $a$, and shares the same beliefs about the distribution of $\theta$ with the principal and agent), any income insurance not provided in the optimal contract between principal and agent will not be forthcoming from any but a risk-loving source.

s An alternative formulation of interest would put a lower limit on the ex post utility level of the agent. However, since the principal cannot verify the state of naturc in this model, it is plausible to assume that the courts (or other legal institutions) would also be unable to verify $\theta$. Consequently, any limitation based explicitly upon the state of nature would be inherently unenforceable.

Similarly, because the agent's actions are unobservable, the liability restrictions considered here cannot be of the form considered in $|2.6|$, wherein the extent of the agent's liability depends upon the action (care) that he chooses to take. 


$$
\begin{aligned}
& \text { subject to: } \quad \text { (i) } a\langle S, \theta\rangle=\underset{a^{\prime} \in A}{\operatorname{argmax}}\left\{S\left[X\left(a^{\prime}, \theta\right)\right]-V\left(a^{\prime}\right)\right\}, \\
& \text { (ii) } S[X(a, \theta)] \geqslant L \quad \forall a \in A \text { and } \forall \theta, \\
& \text { (iii) } E_{\theta}\{S[X(a, \theta)]-W(x, \theta)\} \geqslant U^{0}, \\
& \text { (iv) } X(a, \theta) \geqslant 0 \quad \forall \theta \text { and } \forall a \in A .
\end{aligned}
$$

In the formulation of (PA), $A$ is the set of admissible actions and $E$ is the expectations operator (over $\theta$ ). As defined in the self-selection constraint (i), $a\langle S, \theta\rangle$ is the action that the agent will take in order to maximize his utility after he is presented with a contract and observes the state of nature. This action is assumed to be unique. ${ }^{6}$ The limited liability constraint (ii) can be interpreted (when $x=0$ ) as a specification of the maximum fine that can be imposed upon the agent for failure to put forth any effort. (Note that $W(x(0, \theta), \theta)=W(0, \theta)=0 \forall \theta$.) The individual rationality constraint (iii) restricts the class of contracts under consideration here to those that will necessarily be accepted by the agent (since, more generally, the agent always has the option to refuse to contract at all). Again, Property (5) ensures that there will always be potential gains to both parties from contracting.

Before the properties of the limited liability contract that the principal will offer to the agent are discussed, a brief comparison of (PA) to other models in the literature is offered here. To begin with, the fact that the principal must design an incentive scheme for the agent in the absence of complete information about either the true state of nature or the agent's actions distinguishes (PA) as a "principal-agent" problem as developed by Ross $[18,19]$, Harris and Raviv $[10,11]$, Holmstrom [13], Shavell [23], and Grossman and Hart [9]. Furthermore, the nature of the information asymmetry implicit in (PA) is identical to that considered by Harris and Raviv [10]. It differs from the asymmetry considered in $[9,13,23]$ because in the present study, the agent observes the true state of nature before he chooses an action. The uncertainty in (PA), though, is similar to that analyzed in all of the aforementioned studies because the principal and agent share identical beliefs about the true state of nature when they consummate an agreement to govern their future interaction. It is in this respect that (PA) differs from the models of Green [7], Green and Stokey [8], and Sappington [21, 22], all of which explicitly consider precontractual information asymmetry.

It should also be noted that for the particular case of zero-liability contracts discussed in Sections 3 and 4, the principal-agent relationship

\footnotetext{
${ }^{6}$ In the event that the agent is indifferent among two or more actions, it is assumed (here and throughout the principal-agent literature) that he will select the one most preferred by the principal.
} 
briefly examined in [12] is a special case of the relationship captured in (PA). This point will be developed further below. ${ }^{7}$

Models in the optimal-tax and price-discrimination literature also exhibit structural similarities to (PA). In these models, the distribution of consumer characteristics (which is usually assumed to be continuous) is known by the government $([16])$ or by the discriminating monopolist $([5,24,25])$, but the actual characteristic of any particular individual cannot be observed. It is then the task of the government (monopolist) to design a tax scheme (revenue schedule) based solely on the observable income (purchases) of consumers in order to maximize social welfare (profit). The analogies to (PA) seem apparent.

There are a few studies which, like the present one, assume that the distribution of the state of nature is discrete. Inasmuch, these studies more closely parallel the present one. Notable among these studies is that of Chiang and Spatt [3]. Despite some fundamental differences between models, many of the properties of the optimal (set of) contract(s) in their study are analogous to the properties of the optimal limited liability contract described below. It is also the case that many of the properties of the optimal insurance scheme described in [25] for the case in which $\theta$ may take on one of only two values have their counterpart in the simple zero-liability contract illustrated in Fig. 2 in the following section.

\section{The Structure of Zero-Liability Contracts}

The solution to (PA) is most easily derived by solving the following equivalent problem $\left(\mathrm{PA}^{\prime}\right)$ :

\footnotetext{
${ }^{7}$ It should also be noted here that although (PA) formally captures the interaction between two risk-neutral parties, the formulation does admit another interpretation. If the agent is riskneutral over all payoffs that exceed $L$, but associates infinite disutility with any payoff below $L$, then the limited liability constraint (ii) is simply a necessary condition for the agent's expected utility under the optimal contract to (weakly) exceed his reservation level of expected utility (assuming $x_{n}^{*}$ to be finite). Thus, for an agent who exhibits this type of infinite risk aversion, (PA) and the "standard" formulation of the principal-agent problem are equivalent.

Consequently, the finding that the risk-neutral principal bears all of the risk associated with payoffs below $L$ in the solution to (PA) (see Theorem 1) is consistent with the work of Shavell [1979]. In addition, the results presented in Section 5 are sufficient to prove that the expected utility of the principal varies inversely with $L$ (the "point" of infinite risk aversion for the agent). Hence, the principal would prefer, other things equal, to contract with that agent for whom $L$ is smallest. This finding is similar in nature to Ross' $[20]$ conclusion that when permitted to choose among "public agents," the principal will prefer to contract with that agent whose degree of risk aversion is (in a sense defined precisely by Ross) most similar to his own.
} 


$$
\begin{array}{lll}
\underset{x_{i}, s_{i}}{\operatorname{Maximize}} & & \\
\text { subject to: } & \text { (i) } S_{i=1}^{n} p_{i}\left[x_{i}-S_{i}\right] & \\
& \text { (ii) } \left.\quad x_{i}, \theta_{i}\right) \geqslant S_{j}-W\left(x_{i}, \theta_{i}\right) \geqslant L & \forall i, j, \\
& \text { (iii) } \quad \sum_{i=1}^{n} p_{i}\left[S_{i}-W\left(x_{i}, \theta_{i}\right)\right] \geqslant U^{0}, \\
& \text { (iv) } x_{i} \geqslant 0,
\end{array}
$$

where $x_{i}$ is the value of output produced by the agent in state $\theta_{i}$ and $S_{i}$ is the associated compensation. The equivalence of $(\mathrm{PA})$ and $\left(\mathrm{PA}^{\prime}\right)$ is discussed in Appendix A, and is a direct extension of the work on direct mechanisms contained in [12].

For the purposes of expositional and analytic convenience, the solution to (PA) will be explored in this section and the following one for the special case in which $L=U^{0}$. Such a contract will be referred to as a "zeroliability" contract because the agent has nothing to lose if he accepts it. The zero-liability contract mandates that even if the agent decides to put forth no effort after he observes the state of nature, the principal must compensate the agent with a payment equal in magnitude to his expected return had he decided not to contract with the principal at all, and instead chose autarky. Thus if, for example, the risk-neutral agent were willing to accept any contract on which he expected to break even (i.e., $U^{0}=0$ ), a zero-liability contract would require that the agent not be charged a fee if, after observing $\theta$, he chooses autarky rather than continuing in the employ of the principal. In Section 5, it is shown that the results derived below do not change qualitatively when a wide range of values for $L$ are admitted.

Because a primary focus of this research is to determine whether the principal will offer the agent a first-best contract in the presence of limitations on the liability of the agent, it is of interest here to examine that zero-liability contract which, among all first-best such contracts, is most preferred by the principal. Note that the contract $S(x)=x$ is a first-best, zero-liability contract that will be accepted by the agent as long as the expected surplus from efficient production exceeds the agent's reservation level of expected utility, i.e., as long as $k>0$, a condition that is assumed to hold throughout this work. However, because the agent receives the entire value of any output produced and the principal's payoff is identically zero under this contract, it is not surprising that the principal will never offer this feasible, first-best, zero-liability contract to the agent, as Proposition 1 indicates.

Proposition 1. Among all feasible zero-liability contracts that are also first-best, the one that is most preferred by the principal 
(a) has the agent produce (without compensation) zero output in all (of the lowest) states for which $W_{x}(0, \theta) \geqslant 1$.

(b) leaves the agent indifferent between autarky and producing $x_{m}^{*}$ in the lowest states, $\theta_{m}$, for which $W_{x}\left(0, \theta_{m}\right)<1$.

(c) leaves the agent indifferent in any state $\theta_{i}>\theta_{m}$ between producing $x_{i}^{*}$ and producing $x_{i-1}^{*}$.

The proof of Proposition 1 is relegated to Appendix B.

In order to illustrate the contract described in Proposition 1, a numerical example along the lines of the special case examined in [12] is illustrated in Fig. 1. Here, $n=2, \theta_{1}=1, \theta_{2}=2, U^{0}=0$, and $W(x, \theta)=(x / \theta)^{2}$, so that $x_{1}^{*}=\frac{1}{2}$ and $x_{2}^{*}=2$. In the least productive state, $\theta_{1}$, the agent receives no surplus from production as $S\left(x_{1}^{*}\right)$ is set at $\frac{1}{4}$, the level of disutility incurred in the production of $x_{1}^{*}$. In state $\theta_{2}$, however, the disutility to the agent from producing $x_{1}^{*}$ is only $\frac{1}{16}$. Therefore, in order to induce the agent to produce $x_{2}^{*}$ instead of $x_{1}^{*}$ when $\theta_{2}$ occurs, $S\left(x_{2}^{*}\right)$ must be set in excess of $W\left(x_{2}^{*}, \theta_{2}\right)$ $(-1)$ by the amount of the surplus the agent could realize if he produced $x_{1}^{*}$, i.e., $S\left(x_{1}^{*}\right)-W\left(x_{1}^{*}, \theta_{2}\right)=\frac{3}{16}$. Thus, the least-costly method by which the principal can ensure that an efficient outcome will be realized in both states of nature without violating the agent's zero-liability status is to offer the agent the contract that consists of allocations $A$ and $B$ in Fig. 1.

The agent's state-dependent preferences are illustrated in Figure 1 by two representative indifference curves labelled $\bar{U}^{4}\left(\cdot \mid \theta_{i}\right)$ for each state $\theta_{i}, i=1,2$. The agent's utility increases with movements in a northwesterly direction. The shape of the indifference curves is determined by the assumed form of $W(x, \theta)$ which satisfies Properties (1) through (6). Note that the agent is indifferent between $A$ and autarky in state $\theta_{1}$, and between $A$ and $B$ in state

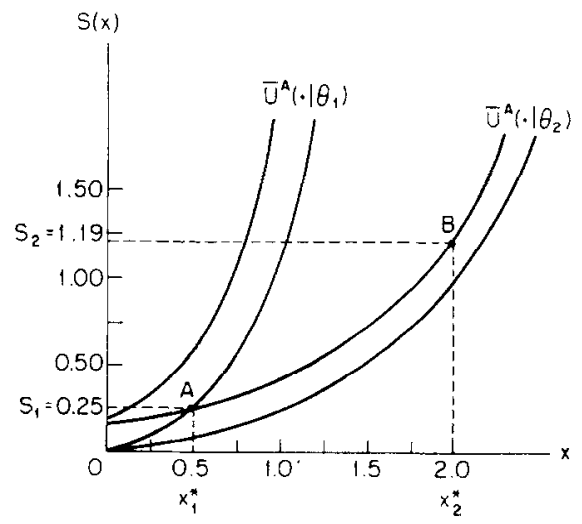

FIG. 1. The principal's preferred first-best zero-liability contract $(A, B)$. 
$\theta_{2}$. Although not shown, the principal's indifference curves are a series of parallel lines with slope of unity. The principal's utility increases to the southeast in Fig. 1.

The "continuous-state indifference" (CSI) structure of this contract (so named because the agent's ex post utility level is the same in state $\theta_{i}$ whether he actually produces $x_{i}$ or the output, $x_{i-1}$, that he would produce if $\theta_{i-1}$ were the true state) corresponds closely to results presented by other authors. Harris and Townsend [12], for example, arrive at identical conclusions for the principal-agent problem that they consider. Their model, in which the agent knows the value of $\theta$ before contracting with the principal, is formally equivalent to the model considered here with $L=U^{0}$ (since a maximum liability level equal to the agent's reservation level of expected utility ensures that the agent will never be worse off than in autarky, which is also the case in the model of Harris and Townsend). The optimal insurance scheme discussed in $[25]$ in which two states (consumer types) are permitted, as well as the optimal set of "time-price" contracts analyzed in $[3]$ also exhibit this general property. It is perhaps not surprising, therefore, that the solution to (PA) with $L=U^{0}$, or the zero-liability contract, that maximizes the principal's expected utility, also has this same structure. The properties of this contract are described more fully in Theorem 1.

THEOREM 1. The zero-liability contract that maximizes the expected utility of the principal

(a) consists of $k+1$ distinct allocations; $k(\leqslant n)$ positive allocations (i.e., $\left(x_{i}, S_{i}\right)$ pairs with $\left.x_{i}>0\right)$ and autarky $(0,0)^{8}$;

(b) has $x_{i}$ (and therefore $S_{i} \equiv S\left(x_{i}\right)$ ) non-decreasing in $\theta$;

(c) extracts all of the surplus from positive production from the agent in (only) the lowest state of nature in which positive production is induced, and promises the agent no payoff in any lower state;

(d) exhibits the same CSI structure as the first-best contract described in Proposition 1;

(e) induces the agent to produce the efficient value of output only in the highest state of nature and in those (lowest) states for which

${ }^{8}$ More precisely, the zero-liability contract that the principal will select need not always explicitly include autarky. For example, the contract illustrated in Fig. 2 consists only of allocations $A^{\prime}$ and $B^{\prime}$. However, the inclusion of $(0,0)$ would not alter the expected utility of either principal or agent. Consequently, because there is no loss in generality if autarky is always included in the zero-liability contract chosen by the principal, this convention is maintained for analytic convenience.

It should also be noted here that the agent can be dissuaded from producing an output other than one of the $k+1$ levels called for under the contract by offering him the least possible compensation, $L$, for any such production. 
$W_{x}(0, \theta) \geqslant 1$. In all other states, the value of output produced by the agent will fall short of its efficient level. ${ }^{9}$

Proof of Theorem 1. With $L=U^{0}$, the individual rationality constraint (iii) is not binding, so that the Lagrangian function associated with $\left(\mathrm{PA}^{\prime}\right)$ is

$$
\begin{aligned}
\mathscr{L}= & \sum_{i=1}^{n} p_{i}\left[x_{i}-S_{i}\right]+\sum_{i=1}^{n} \sum_{\substack{j=1 \\
j \neq i}}^{n} \beta_{i j}\left[S_{i}-W\left(x_{i}, \theta_{i}\right)-S_{j}+W\left(x_{j}, \theta_{i}\right)\right] \\
& +\sum_{i=1}^{n} \gamma_{i}\left[S_{i}-W\left(x_{i}, \theta_{i}\right)-U^{0}\right] .
\end{aligned}
$$

After some simplification, the necessary conditions for a maximum can be shown to include:

$$
\begin{gathered}
p_{i}+\sum_{\substack{j=1 \\
j \neq i}}^{n} \beta_{j i}=\gamma_{i}+\sum_{\substack{j=1 \\
j \neq i}}^{n} \beta_{i j}, \\
p_{i}\left[1-W_{x}\left(x_{i}, \theta_{i}\right)\right]+\sum_{\substack{j=1 \\
j \neq i}}^{n} \beta_{j i}\left[W_{x}\left(x_{i}, \theta_{j}\right)-W_{x}\left(x_{i}, \theta_{i}\right)\right] \leqslant 0 \\
x_{i}[\cdot]=0 .
\end{gathered}
$$

Condition (b) follows from an argument by contradiction. If $x_{i}<x_{j}$ for some $i>j$, then since $S_{j}-S_{i} \leqslant W\left(x_{j}, \theta_{i}\right)-W\left(x_{i}, \theta_{i}\right)$ by the $(i, j)$ th self-selection constraint, $S_{j}-S_{i}<W\left(x_{j}, \theta_{j}\right)-W\left(x_{i}, \theta_{j}\right)$ by Property (3), which violates the $(j, i)$ th self-selection constraint.

The remainder of the proof is outlined here for the case in which $k=n$. The more general proof is more complicated and tedious, but employs the same techniques outlined below.

Employing techniques analogous to those used to prove Proposition 1, it can be shown that for each $i=1, \ldots, n \beta_{i j}=0 \quad \forall j>i+1$ and $\forall j<i-1$. Furthermore, it can be shown that if $\beta_{i, i-1}>0$, then $\beta_{i-1, i}=0 \forall i=2, \ldots, n$.

The proof of Proposition 1 also discusses the arguments which reveal that $\gamma_{1}=1$ and $\gamma_{i}=0 \forall i>1$. This proves condition (c) by the complementary slackness condition associated with the limited liability constraints. Hence, from (1.1),

$$
\beta_{21}=1-p_{1}+\beta_{12}>0,
$$

which implies that $\beta_{21}=1-p_{1}$.

\footnotetext{
${ }^{9}$ In particular, the agent may be induced to produce zero output in some states for which $W_{x}(0, \theta)<1$.
} 
An induction argument then reveals that

$$
\beta_{i, i-1} \doteq 1-\sum_{k=1}^{i-1} p_{k} \quad \forall i=2, \ldots, n,
$$

which proves condition (d). And, since $\beta_{n, n-1}>0, \beta_{j, n}=0 \forall j$, so that $W\left(x_{n}, \theta_{n}\right)=1$, which proves the first statement in condition (e). ${ }^{10}$

Finally, from (1.2),

$$
p_{i}\left[1-W_{x}\left(x_{i}, \theta_{i}\right)\right]=\left[1-\sum_{k=1}^{i} p_{k}\right]\left[W_{x}\left(x_{i}, \theta_{i}\right)-W_{x}\left(x_{i}, \theta_{i+1}\right)\right] .
$$

Hence, since the right-hand side of this equation is strictly positive by Property (3), $W_{x}\left(x_{i}, \theta_{i}\right)<1$ which, by Property (2), proves condition (e).

Q.E.D.

The zero-liability contract that maximizes the expected utility of the principal is depicted in Figure 2 for the special case illustrated in Fig. 1. Here, $\theta_{1}$ and $\theta_{2}$ are assumed equally likely to occur. As indicated by condition (e) of Theorem 1, the main difference to note is that this contract (consisting of points $A^{\prime}$ and $B^{\prime}$ ) will now induce the agent to produce an inefficiently small output $x_{1}=\frac{2}{7}<\frac{1}{2}=x_{1}^{*}$ when $\theta_{1}$ occurs. Note also, though, that the payment to the agent for producing the efficient value of output (2) when $\theta_{2}$ occurs is only $52 / 49$, which is less than the payment (19/16) awarded the agent for such production under the first-best limited liability contract most preferred by the principal. Herein lies the advantage to the principal of intentionally inducing inefficient production when compelled to respect limitations on the agent's liability. This advantage is developed more completely in the following section.

\section{Comparative Statics and an Explanation of the Findings}

It was noted in Section 3 that the derivation of the zero-liability contract most preferred by the principal when there are only two states of nature is formally equivalent to the problem considered in [12]. Therefore, it is perhaps not surprising that two important conclusions of the present study are not at all dissimilar to the observations of Harris and Townsend. First, the authors essentially prove that $x_{1}<x_{1}^{*}$ as illustrated in Fig. 2. Theorem 1 is the generalization of this finding (and the results in Section 4 further

${ }^{10}$ In the more complete proof of Theorem 1 (i.e., where $k$ may be strictly less than $n$ ), it is first necessary to prove that $x_{n-1}<x_{n}$ before one can conclude that $x_{n}=x_{n}^{*}$. An outline of this proof is presented in Appendix B. 


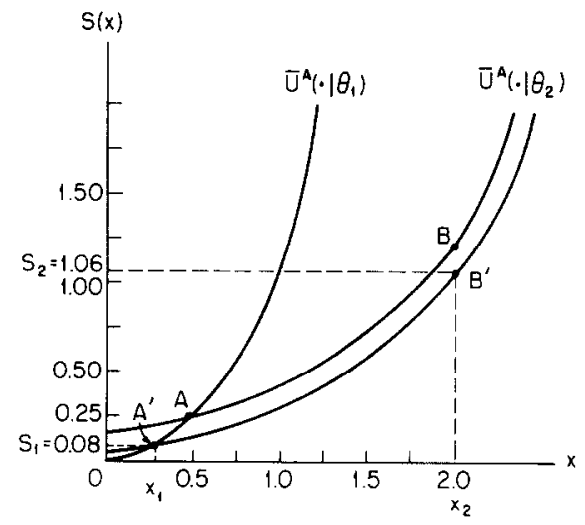

FIG. 2. The principal's preferred zero-liability contract $\left(A^{\prime}, B^{\prime}\right)$.

generalize the observation). Second, the authors essentially point out for their two-state example that although the structure of the first-best contract described in Proposition 1 does not depend upon the principal's beliefs, the value of the output that the agent will be induced to produce in any state when the principal is not restricted to first-best contracts will generally depend upon these prior beliefs. It is the purpose of this section to define this dependence precisely, and then to employ the findings derived here to more fully explain the structure of the limited liability contract derived in Theorem 1.

Proposition 2 analyzes the manner in which the zero-liability contract most preferred by the principal depends upon the principal's beliefs about the distribution of $\theta$. The proposition makes use of the following notation:

$$
\begin{aligned}
\Theta^{i}= & \text { the set of all } \theta_{j} \text { for which the agent produces } x_{i}^{\prime} \\
& \text { under the contract described in Theorem } 1, \\
& i=0,1, \ldots, k, \text { where } x_{0}^{\prime}=0, \\
I^{i}= & \text { the set of numerical subscripts on those } \theta_{j} \in \Theta^{i}, \\
\theta_{i}^{\mathrm{min}}= & \text { minimum }\left\{\Theta^{i}\right\}, \text { and } P^{i}=\sum_{j \in I^{i}} p_{j}
\end{aligned}
$$

Proposition 2. Let $x_{0}^{\prime}<x_{1}^{\prime}<\cdots<x_{k}^{\prime}$ be the $k(\leqslant n)$ distinct, positive values of output that along with autarky $\left(x_{0}^{\prime}\right)$ constitute the solution to $(P A)$. If Properties (1) through (6) are satisfied, the following comparative static results hold:

(i) $x_{i}^{\prime}$ increases as $p_{j}\left(j \in I^{i}\right)$ increases and $p_{h}\left(h \in I^{z}, i<z \leqslant k\right)$ decreases by a corresponding magnitude. 
(ii) $x_{i}^{\prime}$ increases as $p_{j}\left(j \in I^{i}\right)$ increases and $p_{h}\left(h \in I^{z}, 0 \leqslant z<i<k\right)$ decreases by a corresponding magnitude.

(iii) $x_{i}^{\prime}$ increases as $p_{j} \quad\left(j \in I^{z}, 0 \leqslant z<i\right)$ increases and $p_{h}$ ( $h \in I^{y}, i \leqslant y<k$ ) decreases by a corresponding magnitude.

(iv) $x_{i}^{\prime}$ is unaffected when $p_{j}\left(j \in I^{i}\right)$ increases and $p_{j}^{\prime}\left(\neq p_{j}\right)$ decreases by a corresponding magnitude.

Proof of Proposition 2. The more general counterpart to equation (1.3) in the proof of Theorem 1 can be shown to be

$P_{i}\left[1-W_{x}\left(x_{i}^{\prime}, \theta_{i+1}^{\min }\right)\right]=\left(1-\sum_{r=0}^{i-1} P_{r}\right)\left[W_{x}\left(x_{i}^{\prime}, \theta_{i}^{\min }\right)-W_{x}\left(x_{i}^{\prime}, \theta_{i+1}^{\min }\right)\right]$.

This equation can be rearranged to prove that $B\left(x_{i}^{\prime}\right)=\left[P_{i}\right] /\left[1-\sum_{r=0}^{i-1} P_{r}\right]$, where $B\left(x_{i}^{\prime}\right)$ is defined to be the ratio of $\left\{W_{x}\left(x_{i}^{\prime}, \theta_{i}^{\min }\right)-W_{x}\left(x_{i}^{\prime}, \theta_{i+1}^{\min }\right)\right\}$ to $\left\{1-W_{x}\left(x_{i}^{\prime}, \theta_{i+1}^{\min }\right)\right\}$.

The derivative of $B(\cdot)$ with respect to $x_{i}^{\prime}, B^{\prime}\left(x_{i}^{\prime}\right)$, can be shown to be strictly positive using Properties (3), (4) and (6) and condition (e) of Theorem 1. Now

$$
\begin{aligned}
d B\left(x_{i}^{\prime}\right) / d p_{j}= & {[1] /\left[1-\sum_{r=0}^{i-1} P_{r}\right] \quad \text { for } j \in I^{i}, } \\
d B\left(x_{i}^{\prime}\right) / d p_{h}= & 0 \quad \text { for } h \in I^{z} \text { where } i<z \leqslant k, \\
d B\left(x_{i}^{\prime}\right) / d p_{h}= & {\left[P_{i}\right] /\left[1-\sum_{r=0}^{i-1} P_{r}\right]^{2}=B\left(x_{i}^{\prime}\right)\left[d B\left(x_{i}^{\prime}\right) / d p_{j}\right] } \\
& \text { for } h \in I^{2} 0 \leqslant z<i, \text { and } j \in I^{i} .
\end{aligned}
$$

Results (i) through (iv) then follow from these derivatives, noting that $B\left(x_{i}^{\prime}\right) \leqslant 1 \forall i$ and that since $B^{\prime}\left(x_{i}^{\prime}\right)>0$, any increase in $B(\cdot)$ corresponds to an increase in $x_{i}^{\prime}$ for constant values of $\theta_{i}^{\min }$ and $\theta_{i+1}^{\min }$.

Q.E.D.

Proposition 2 provides the missing link to a complete understanding of the limited liability contract that maximizes the expected utility of the principal. Consider the implications of Proposition 2 for the simple example illustrated in Fig. 2. As was noted above, if he designs the contract such that the agent is compensated for producing an inefficiently small output in the lower state of nature (i.e., if he sets $x_{1}<x_{1}^{*}$ ), the principal reduces the magnitude of the payment needed to induce a higher level of output $\left(x_{2}^{*}\right)$ in the more productive state, $\theta_{2}$. And more generally, when designing a limited liability contract, the principal weighs the expected benefits of setting $x_{i}$ below $x_{i}^{*}$ (benefits which accrue in the event that some state above $\theta_{i}$ is realized) against the costs of inefficiency (costs which are borne if $\theta_{i}$ is realized). 
Thus, as states above $\theta_{i}$ become more likely and $\theta_{i}$ itself less likely, ceteris paribus, $x_{i}$ will be set further below $x_{i}^{*}$ in the contract most preferred by the principal. ${ }^{11}$ In the example in Fig. 2 , the greater is $p_{2}$ relative to $p_{1}$, the smaller will be the value of $x_{1}$.

It should be noted, too, that because the benefits associated with inducing an inefficient outcome in any state are realized only when higher states of nature occur, there are no incentives for the principal to induce an inefficient outcome in the highest state of nature. Furthermore, the distribution of $\theta$ and the technology may be such that in some states, the expected benefits of elevating $x_{i}$ above the level of $x_{i-1}$ in the contract selected by the principal are outweighed by the expected costs. Under such circumstances, $x_{i}$ and $x_{i-1}$ will coincide and the limited liability contract offered to the agent will be a "pooling" contract in the terminology of Stiglitz [25].

Finally, it should be emphasized why the foregoing concerns are relevant only in the presence of limited liability restrictions. Absent any floor on the payoff to the risk-neutral agent, any rent that the agent may gain when the principal expands $x_{i}$ to its efficient level in each state $\theta_{i}$ can be effectively negated by demanding that the agent pay a larger lump sum payment in order to contract at all. Consequently, it is only when limited liability constraints are binding that social efficiency and private utility maximization for the principal are not coincidental.

\section{Generalization of the Results}

The findings in Section 3 and 4 were derived under the assumption that $L=U^{0}$, so that the individual rationality constraint ((iii) in (PA)) imposed no restrictions on the principal's choice of a contract beyond those imposed by the limited liability constraints. In this section, it is demonstrated that the qualitative results derived above are unchanged when more general values of $L$ are admitted. It is also shown that the principal will offer the agent a firstbest contract if $L$ is sufficiently small.

Whenever the legal extent of the agent's liability falls short of his reservation level of expected utility (i.e., whenever $L \geqslant U^{0}$ ), the individual rationality constraint (iii) in (PA) is not binding. Under such circumstances, therefore, the techniques outlined above can be directly employed to show that the limited liability contract most preferred by the principal has the same properties as the zero-liability contract described by Theorem 1 and

$"$ Of course, a contract that is not first-best provides strictly less total expected surplus than does a first-best contract. However, the first-order effect on the total surplus of a deviation from the first-best contract is zero since the total surplus in any state $\theta_{i}$ is $x_{i}-W\left(x_{i}, \theta_{i}\right)$, and the derivative of this expression with respect to $x_{i}$ when evaluated at $x_{i}^{*}$ is identically zero. Consequently, some deviation from the first-best contract will always be pursued by the principal when the limited liability constraints are binding. 
Eq. (2.1) in the proof of Proposition 2. The only difference is a shift in the "origin" of the contract, so that in the lowest state in which positive production takes place, the agent's compensation leaves him indifferent between carrying out such production and providing no effort in return for a payment of $L$.

Similarly, for values of $L$ that are less than but "close" to $U^{n}$, the zeroliability contract described in Theorem 1 and Eq. (2.1) (with an appropriate shift in the origin of the contract) may provide non-negative expected utility to the agent, and will therefore be chosen by the principal for these smaller values of $L$. However, for $L$ sufficiently smaller than $U^{0}$, the aforementioned zero-liability contract (with shifted origin) may not provide the agent with a level of expected utility that exceeds his reservation level. Consequently, in order to induce the agent to become party to the contract, the principal must transfer some expected surplus to him. The best way to do so from the principal's point of view is, loosely speaking, to maintain the CSI structure of the zero-liability contract but increase the level of each $x_{i}$ towards $x_{i}^{*}$. This procedure increases the surplus that the principal expects to award the agent, but is preferable to granting the agent a simple lump-sum bonus (in some or all states of nature) because it induces additional output from the agent in each state in return for payments which are less than the value of the output to the principal. The more binding is the individual rationality constraint at the optimum, the smaller is the discrepancy between each $x_{i}$ and the corresponding $x_{i}^{*}$ in the optimal contract, and thus the "closer" is the contract that the principal will design to a first-best contract. For $L$ sufficiently far below $U^{0}$, the two will coincide.

To make those observations more precise, let $\phi$ represent the Lagrange multiplier associated with the individual rationality constraint (iii) in $\left(\mathbf{P A}^{\prime}\right)$. It can be shown that at the optimum, $0 \leqslant \phi \leqslant 1$, and $\phi$ is larger the smaller is $L$, ceteris paribus. $\phi=0$ corresponds to the situation in which the solution to $\left(\mathrm{PA}^{\prime}\right)$ is the zero-liability contract described in Theorem 1 with the appropriate shift in its origin. $\phi=1$ corresponds to the situation in which the individual rationality constraint is the only constraint that is binding at the optimum. In the latter case, because the agent is risk-neutral, the principal will select a first-best contract. Among the solutions to $\left(\mathrm{PA}^{\prime}\right)$ when $\phi=1$ is a first-best contract of the general form described in Proposition 1, but where the agent's ex post utility level in the least productive state is sufficiently large $(\geqslant L)$ to ensure that the agent's expected utility under the contract is identically $U^{0} .{ }^{12}$

12 The lump sum contract $S(x)=x-k$ that provides the agent with only his reservation level of expected utility is also a solution to ( $\left.\mathrm{PA}^{\prime}\right)$ when $\phi=1$. This lump sum contract would constitute the unique solution to an analogous problem in which the principal was risk averse. 
For those cases in which $0<\phi<1$ (i.e., whenever some limited liability constraint is binding at the optimum as well as the individual rationality constraint), the solution to $\left(\mathrm{PA}^{\prime}\right)$ has the usual CSI structure, leaves the agent's ex post utility level at $L$ in the lowest state for which positive production is induced, and (using the notation which precedes Proposition 2) has each of the $k$ distinct, positive output levels, $x_{i}^{\prime}$, determined by the equation:

$$
\begin{aligned}
P_{i}- & {\left[1-\sum_{r=0}^{i-1} P_{r}\right] W_{x}\left(x_{i}^{\prime}, \theta_{i}^{\mathrm{min}}\right)+\left[1-\sum_{r=0}^{i} P_{r}\right] W_{x}\left(x_{i}^{\prime}, \theta_{i+1}^{\mathrm{min}}\right) } \\
=\phi & {\left[1-\sum_{r=0}^{i} P_{r}\right]\left[W_{x}\left(x_{i}^{\prime}, \theta_{i+1}^{\min }\right)-W_{x}\left(x_{i}^{\prime}, \theta_{i}^{\mathrm{min}}\right)\right] . }
\end{aligned}
$$

Using techniques analogous to those employed in the proof of Theorem 1, it can be shown that whenever $\phi<1$ at the optimum, $x_{i}$ will fall short of $x_{i}^{*}$ in all states of nature for which $W_{x}(0, \theta)>1$ except the very highest (where the two coincide).

Thus, the qualitative results discussed in Sections 3 and 4 are largely unaltered by the introduction of more general liability limits. Efficiency with strictly positive output is attained only in the highest state of nature under the limited liability contract selected by the principal when $L$ is sufficiently large relative to the agent's reservation level of expected utility. As $L$ becomes smaller and smaller relative to this benchmark, though, the value of output produced in every state under the limited liability contract most preferred by the principal approaches its efficient level, until efficiency is achieved in every state when the liablity constraints are no longer binding.

\section{Conclusions}

The main thrust of this research has been to show that when the principal is compelled to respect the limited liability status of the risk-neutral agent, the principal will generally not offer the agent a first-best contract. The form of the limited liability contract that the principal will design was derived and explained in detail.

In closing, a few additional issues are raised. First, it has been assumed throughout that the relationship between principal and agent was an exclusive one. The presence of pre-contract competition among agents, though, is unlikely to be sufficient to guarantee that a first-best contract will ultimately be realized between the principal and the "winning" agent. If, for example, the state of nature can only be observed after specialized plant and equipment has been installed and production has begun, it may be necessary 
(and socially optimal in order to avoid duplication of facilities) for the principal to contract with only a single agent. And although the principal may benefit from initial bidding among agents for the right to produce, the final limited liability contract signed will be of the form derived above, and therefore generally not first-best. It is only if there is significant competition among identical agents, each of whom knows the actual realization of $\theta$ before contracting with the principal (contrary to the scenario considered here) that an outcome which is ex post Pareto efficient will be ensured.

Second, it should be noted that throughout the foregoing analysis the agent was assumed to be risk-neutral. If the agent were risk averse, however, the qualitative results reported in Theorem 1 would be unlikely to change. Instead, there would be an additional reason for the principal to choose other than a first-best contract; namely, to take advantage of the risk-sharing properties offered by contracts that are not first-best (properties discussed in, for example, $|13,23|)$.

Finally, because the principal and agent were assumed here to share symmetric beliefs about the distribution of $\theta$ before a contract is agreed upon, some important complications were omitted from the analysis. In particular, in the absence of symmetric precontractual beliefs, the principal and agent will not necessarily agree upon whether any particular contract (of the limited liability variety or otherwise) provides a level of expected utility for the agent that exceeds his reservation level. An analysis of this particular complication and other related ones can be found in [21, 22].

\section{APPENDIX A}

A brief outline of the proof of the equivalence of $(\mathrm{PA})$ and $\left(\mathrm{PA}^{\prime}\right)$ is presented here.

I. Prove that any solution to (PA) is a solution to (PA).

A. Let $\bar{S}(x), \bar{a}\langle\bar{S}, \theta\rangle$, and $\bar{x}=X(\bar{a}, \theta)$ solve (PA).

B. Show that $\bar{S}(x)$ and $\bar{x}$ satisfy the constraints in ( $\left.\mathrm{PA}^{\prime}\right)$. Here, it is important to note that since the payment to the agent must never fall below $L$ regardless of the output produced, were his ex post utility ever to fall below $L$, the agent would not be acting rationally as he could always do better if he were to supply no effort.

C. Prove, by contradiction, that $\bar{S}(x)$ and $\bar{x}$ maximize the objective function in ( $\left.\mathrm{PA}^{\prime}\right)$, utilizing the fact that they maximize the objecitve function in (PA).

II. Prove that any solution to $\left(\mathrm{PA}^{\prime}\right)$ is a solution to (PA). The proof is analogous to that outlined in $\mathbf{I}$. 
III. Conclusion: Since any solution to (PA) is a solution to $\left(\mathbf{P A}^{\prime}\right)$, and any solution to $\left(\mathrm{PA}^{\prime}\right)$ is a solution to (PA), the two problems are equivalent.

\section{APPENDIX B}

Proof of Proposition 1. The form of the zero-liability contract which, among all first-best contracts, is preferred by the principal is derived by minimizing $\sum_{i=1}^{n} p_{i} S_{i}$ subject to constraints (i) through (iv) in ( $\left.\mathrm{PA}^{\prime}\right)$, with $L$ set equal to $U^{0}$ and $x_{i}$ equal to $x_{i}^{*}$ for all $i=1, \ldots, n$. By definition, the efficient value of output is zero in any state for which the disutility to the agent of producing positive output exceeds the value of that output to the principal. Hence, condition (a) follows.

Since $L=U^{0}$, the individual rationality constraint (iii) is redundant in light of the zero liability constraint (ii), and can be ignored. Considering, now, only the $(n-m+1)$ states for which $x_{i}^{*}>0$, the necessary conditions for a maximum reveal that

$$
p_{i}+\sum_{\substack{j=m \\ j \neq i}}^{n} \beta_{j i}=\gamma_{i}+\sum_{\substack{j=m \\ j \neq i}}^{n} \beta_{i j} \quad \forall i=m, \ldots, n,
$$

where $\beta_{i j}$ is the non-negative Lagrange multiplier associated with the selfselection constraint (i), and $\gamma_{i}$ the corresponding multiplier for the limited liability constraint (ii).

To prove that $\gamma_{i}=0 \forall i>m$ assume the contrary. Then, using the selfselection constraint and Property (1), it follows that

$$
\begin{aligned}
U^{0} & =S_{i}-W\left(x_{i}^{*}, \theta_{i}\right) \geqslant S_{i-1}-W\left(x_{i-1}^{*}, \theta_{i}\right) . \\
& >S_{i-1}-W\left(x_{i-1}^{*}, \theta_{i-1}\right),
\end{aligned}
$$

which is a violation of the limited liability constraint in state $\theta_{i-1}$. Consequently, it follows that $\gamma_{m}=\sum_{k=m}^{n} p_{k}>0$ by summing the above $n-m+1$ necessary conditions. This proves condition (b) using the complementary slackness condition for the limited liability constraint in state $\theta_{m}$.

To prove that $\beta_{i j}=0 \forall j>i+1$ for all $i=m, \ldots, n-2$, assume the contrary.

Then

$$
S_{j}-W\left(x_{j}^{*}, \theta_{i}\right)=S_{i}-W\left(x_{i}^{*}, \theta_{i}\right) \geqslant S_{i+1}-W\left(x_{i+1}^{*}, \theta_{i}\right) .
$$


Consequently,

$$
\begin{aligned}
S_{j}-S_{i \mid 1} & \geqslant W\left(x_{j}^{*}, \theta_{i}\right)-W\left(x_{i+1}^{*}, \theta_{i}\right) \\
& >W\left(x_{j}^{*}, \theta_{i+1}\right)-W\left(x_{i+1}^{*}, \theta_{i+1}\right)
\end{aligned}
$$

by Property (3), or equivalently, $S_{i+1}-W\left({ }_{i+1}^{*}, \theta_{i+1}\right)<S_{j}-W\left(x_{j}^{*}, \theta_{i+1}\right)$, which violates one of the self-selection constraints.

Similar techniques show that $\beta_{i j}=0 \quad \forall j<i-1$ for all $i=m+2, \ldots, n$. Finally, a similar proof by contradiction reveals that if $\beta_{i, i-1}>0$, then $\beta_{i-1, i}=0 \forall i=m, \ldots, n$. And, from (B1.1), $\beta_{m+1, m}=\gamma_{m}-p_{m}+\beta_{m, m+1}>0$, so that $\beta_{m, m+1}=0$ and $\beta_{m+1, m}=\sum_{k=m+1}^{n} p_{k}$.

A straightforward induction argument reveals that $\beta_{i, i-1}=\sum_{k=i}^{n} p_{k}>0$ $\forall i=m+1, \ldots, n$. Hence, by the complementary slackness condition associated with the self-selection constraints, condition (c) of Proposition 1 follows.

Q.E.D.

LEMMA. In an $n$-state world $(n>2)$, the zero-liability contract chosen by the principal has $x_{n}>x_{n-1}$.

Outline of Proof. There are two distinct cases to consider.

Case I. $x_{n-1}=0$. In this case, since $W_{x}\left(0, \theta_{n}\right)<1$ by Property (5), the principal will be strictly better of if he sets $x_{n}>0\left(x_{n} \leqslant x_{n}^{*}\right)$ and $S\left(x_{n}\right)=$ $W\left(x_{n}, \theta_{n}\right)$ rather than having $x_{n}=S\left(x_{n}\right)=0$. Furthermore, the agent's expected utility is unchanged by this alteration and none of the self-selection constraints are violated.

Case IIA. $x_{n-1}>0$ and $x_{n-1}<x_{n}^{*}$. In this case, the principal's expected utility is strictly increased if, instead of setting $x_{n}=x_{n-1}$, he sets $x_{n}=x_{n-1}+\varepsilon\left(\varepsilon \leqslant x_{n}^{*}-x_{n-1}\right)$ and $S_{n} \equiv S\left(x_{n}\right)$ such that $S_{n}-W\left(x_{n}, \theta_{n}\right)=$ $S_{n-1}-W\left(x_{n-1}, \theta_{n}\right)$. Also, such an alteration neither reduces the agent's expected utility nor causes him to change his production decision in any of states $\theta_{1}$ through $\theta_{n-1}$.

Case IIB. $x_{n-1}>0$ and $x_{n-1} \geqslant x_{n}^{*}$. The proof of this case is similar to those discussed above, wherein a new feasible contract that is strictly preferred by the principal is constructed, in which $x_{n-1}=x_{n-1}^{*}$ and $x_{n}=x_{n}^{*}$.

Q.E.D.

\section{REFERENCES}

1. J. H. Barton, The economic basis of damages for breach of contract, J. Leg. Studies 1 (June 1972), 277-304.

2. J. P. Brown, Toward an economic theory of liability, J. Leg. Studies 2 (June 1973). 323-349. 
3. R. Chiang and C. Spatt, "Imperfect Price Discrimination and Welfare," Working Paper No. 44-79-80, Carnegie-Mellon University GSIA, November 1979.

4. P. A. Diamond and E. Maskin, An equilibrium analysis of search and breach of contract, I: Steady states, Bell J. Econ. 10 (Spring 1979), 282-316.

5. M. B. Goldman, H. E. Leland, and D. S. Sibley, "Optimal Nonuniform Prices," Discussion Paper No. 100, Bell Laboratories, May 1977.

6. J. R. Green, On the optimal structure of liability laws, Bell J. Econ. 7 (Autumn 1976), 553-574.

7. J. R. GREEN, "Statistical Decision Theory Requiring Incentives for Information Transfer," Discussion Paper No. 708, Harvard Institute of Economic Research, May 1979.

8. J. R. Green and N. L. Stokey, "A Two-Person Game of Information Transmission," Discussion Paper No. 751, Harvard Institute of Economic Research, March 1980.

9. S. Grossman and O. Hart, "An Analysis of the Principal-Agent Problem," Working Paper No. 80-17, University of Pennsylvania, July 1980.

10. M. HaRris and A. Raviv, Optimal incentive contracts with imperfect information, $J$. Econ. Theory 20 (April 1979), 231-259.

11. M. Harris AND A. Raviv, Some results on incentive contracts with applications to education and employment, health insurance, and law enforcement, Amer. Econ. Rev. 68 (March 1978), 20-30.

12. M. HARRIS AND R. M. TOWNSEND, Resource allocation under asymmetric information, Econometrica 49 (January 1981), 33-64.

13. B. Holmstrom, Moral hazard and observability, Bell J. Econ. 10 (Spring 1979), 74-91.

14. L. HuRwiCz AND L. Shapiro, Incentive structures maximizing residual gain under incomplete information, Bell J. Econ. 9 (Spring 1978), 180-192.

15. T. Lewis, Bonuses and penalties in incentive contracting, Bell. Econ. J. 11 (Spring 1980), 292-301.

16. J. A. MirleEs, An exploration in the theory of optimum income taxation, Rev. Econ. Studies 38 (April 1971), 175-208.

17. R. A. PoSner, "Economic Analysis of Law," Little, Brown \& Co., Boston, 1972.

18. S. A. Ross, On the economic theory of agency and the principle of similarity, in "Essays on Economic Behavior Under Uncertainty" (M. S. Balch, D. L. McFadden, and S. Y. Wu, Eds.), pp. 215-237, North-Holland, Amsterdam, 1974.

19. S. A. Ross, The economic theory of agency: The principal's problem, Amer. Econ. Rev. 63 (May 1973), 134-139.

20. S. A. Ross, Equilibrium and agency-inadmissible agents in the public agency problem. Amer. Econ. Rev. 69 (May 1979), 308-312.

21. D. Sappington, "Continuous vs. Discrete Analysis: An Application to the PrincipalAgent Model with Asymmetric Information, "Working Paper, The University of Michigan, May 1981.

22. D. SAPPINGTON, Information asymmetry between orincinal and agent: The continuous case, Econ. Letl. 5 (1980), 371-375.

23. S. ShavelL, Risk sharing and incentives in the principal and agent relationship, Bell J. Econ. 10 (Spring 1979), 55-73.

24. M. SPENCE, Nonlinear prices and welfare, J. Pub. Econ. 8 (August 1977), 1-18.

25. J. E. Stiglitz, Monopoly, non-linear pricing and imperfect information: The insurance market, Rev. Econ, Studies 44 (October 1977), 407-430. 